\title{
Therapeutic Irradiation: Consequences for Bone and Bone Marrow Adipose Tissue
}

\author{
Samantha Costa ${ }^{1,2,3}$ and Michaela R. Reagan ${ }^{1,2,3 *}$ \\ ${ }^{1}$ Center for Clinical and Translational Research, Maine Medical Center Research Institute, Scarborough, ME, United States, \\ ${ }^{2}$ University of Maine Graduate School of Biomedical Science and Engineering, Orono, ME, United States, ${ }^{3}$ Tufts University \\ School of Medicine, Boston, MA, United States
}

\section{OPEN ACCESS}

Edited by:

Nathalie Bravenboer

VU University Medical

Center, Netherlands

Reviewed by:

Eleni Douni,

Agricultural University of

Athens, Greece

Monica De Mattei,

University of Ferrara, Italy

*Correspondence:

Michaela R. Reagan

mreagan@mmc.org

Specialty section:

This article was submitted to

Bone Research,

a section of the journal

Frontiers in Endocrinology

Received: 13 May 2019

Accepted: 09 August 2019

Published: 29 August 2019

Citation:

Costa S and Reagan MR (2019)

Therapeutic Irradiation:

Consequences for Bone and Bone

Marrow Adipose Tissue.

Front. Endocrinol. 10:587.

doi: $10.3389 /$ fendo.2019.00587
Radiotherapy continues to be one of the most accepted medical treatments for cancer. Localized irradiation is the most common treatment for prostate, pancreatic, rectal, cervical and endometrial malignancies. Conventional localized fractions are total doses of 30-62Gy at 1.8-2Gy per fraction, with administration of 60Gy often used for tumor ablation. However, even the lowest dose of localized irradiation exposure can result in adverse complications to adjacent organs, tissues, and vessels, which absorb a portion of the treatment. Skeletal complications are common amongst cancer patients undergoing these localized treatments. Irradiation exposure causes deterioration to the overall quantity and quality of bone by interfering with the trabecular architecture through increased osteoclast activity and decreased osteoblast activity. Irradiation-induced bone damage parallels adipocyte infiltration of the bone marrow (BM) resulting in compositional alterations of the microenvironment that may further affect bone quality and disease state. There may also be direct effects of irradiation on the BM adipocyte/pre-adipocyte, although in vitro findings do not always agree and cellular response is dependent on irradiation dosage. Hematopoietic cells also become apoptotic upon irradiation, which causes a range of skeletal effects. Bone loss leaves patients at a greater risk for osteopenia, osteoporosis, osteonecrosis, and skeletal fractures that drastically reduce quality of life. Osteoanabolic agents stimulate bone formation and reduce fracture risk in patients with low bone density; thus, osteoanabolic or anti-resorptive agents may be useful co-treatments with irradiation. This review discusses these topics and proposes further research directions using novel or combination therapies to enhance bone health during irradiation.

Keywords: irradiation, bone marrow microenvironment, bone marrow adipose tissue, osteoblast, adipocyte

\section{INTRODUCTION}

Since the discovery of the X-ray in 1895 , irradiation science has offered advanced developments in techniques, multidisciplinary approaches, and research (1). Radiation therapy continues to be a widely accepted treatment for malignant cancers through its effective manner of killing cancer cells and reducing tumor size $(1,2)$. Irradiation induces free radicals in the form of reactive oxygen species (ROS) that leads to DNA damage (2). With improved treatment regiments, there have been improvements in disease outcomes and reductions in the adverse side effects of 
irradiation therapy $(1,2)$. The effectiveness of irradiation therapies, in combination with advances in pharmaceutical treatments, early detection, prevention, and cancer awareness, has drastically improved patient quality of life and decreased mortality rates; in some cases, these advancements have changed cancer from being an acute disease to a treatable chronic disease (1). The American Cancer Society projected there were over 2.6 million fewer cancer-related deaths from 1991 to 2016 (3).

Despite the advancements in irradiation therapies, there is still an unmet concern surrounding the systemic and localized effects of irradiation on adjacent tissues, vessels, and bone (46). Patients undergoing irradiation therapy have the potential to experience increased irradiation toxicity, even with fractionation of treatments, and adjacent soft tissue and bone damage as an adverse side effect due to limited tumor uptake and retention of irradiation doses (6). Tumor microenvironments promote tumor growth and angiogenesis through paracrine stimulatory factors and immune-mediated interactions (7). There are many cytokines released by the immune system that are considered "pro-tumor" or "anti-tumor" that alter the tumor microenvironment (7). After irradiation exposure, there are increased inflammatory cytokines, IL- $1 \alpha / \beta$, IL-6, IL-17, TNF$\alpha$, and VEGF, and evidence of increased cellular senescence, demonstrated through increased senescence-associated secretory phenotype (SASP) proteins $(2,7,8)$. This change to the tumor microenvironment and increased immune activity is thought to explain the abscopal effect, in which localized irradiation results in regression of metastatic cancer that are distant from the initial irradiation site (7).

Due to the high calcium content, bone absorb 30-40\% more irradiation than the surrounding tissues; thus, the absorption of any given irradiation dose is considerably higher in bone than the surrounding tissues, making bone a common site for irradiation-induced damage (9). As previously discussed, irradiation exposure releases cytokines as an injury response that triggers acute inflammation $(9,10)$. This acute inflammation is characterized by increased vascular permeability with localized edema, destruction of endothelial cells, and an association with vascular thrombosis (9). Irradiation exposure also induces late stage fibroatrophy that results in poorly vascularized tissue which does not allow for proper healing, ultimately increasing tissue fragility and the recurrence of inflammation upon local injury $(9,10)$.

The damage observed within the bone and bone marrow (BM) after irradiation therapy is similar to the pathological conditions seen with osteoporosis (2). There is a decrease in trabecular bone volume, an increase in bone marrow adiposity (BMA), increased CTX/TRAP5 levels in the serum, and prolonged fracture healing times (2). Irradiation also depletes hematopoietic and skeletal stem cell populations within the BM (11-13). Bone marrow transplants allow for trabecular recovery, reduced BMA, and increased cell number within the BM microenvironment (12). Skeletal stem cells (SSCs), previously referred to as mesenchymal stem cells, appear to be effected by the irradiation source (photon irradiation vs. ionizing irradiation) and dose resulting in the varying differentiation potential observed in different in vitro studies (Figure 1) (11, 14-16). In murine models, the balance favors adipogenesis at the expense of osteogenesis, as a result of irradiation-induced bone loss. This bone loss is in part due to the increased osteoclast activity immediately following irradiation exposure and then the latent decrease in osteoblast activity in the sequential weeks $(4,5,12,17)$.

Studies have shown that osteoanabolic agents stimulate bone formation and reduce fracture risk in patients with low bone density (18-20). Since an adverse side effect of irradiation is decreased bone density and increased bone fragility, combination therapies of osteoanabolic, or anti-resorptive agents may be useful for patients receiving irradiation therapies. This review will discuss these topics and propose further research directions including in vitro and in vivo studies using novel or combination therapies to enhance bone strength in patients after irradiation (18).

\section{IN VITRO IRRADIATION OF SKELETAL STEM CELLS ALTERS DIFFERENTIATION POTENTIAL}

The regenerative capabilities of SSCs in vitro have been shown in a multitude of tissue damage models (14). Human bone marrow skeletal stem cells (hSSCs) have been shown to be resistant to the effects of low-dose irradiation (2.5Gy) with no apparent changes to morphology or immunophenotype (11). Preciado et al. have shown irradiated and non-irradiated hSSCs still expressed CD73, CD90, CD105, CD44, and CD166 and are negative for CD34, CD45, CD14, CD19, and HLA-DR, which are definitive markers of a typical SSC immunophenotypic profile (11). Irradiated hSSCs also had no significant changes to cell viability 1 or $72 \mathrm{~h}$ after exposure compared to non-irradiated hSSC controls (11). However, low-dose irradiation exposure affected hSSC behavior and differentiation potential in vitro (11). Irradiated hSSCs were capable of differentiation, but had significantly less adipocytes, evident through decreased Oil-Red-O staining and significantly less mRNA expression of adipogenic differentiation markers, $C E B P \alpha$ and PPAR $\gamma$, when compared to the non-irradiated cells (11). On the other hand, low level irradiation exposure stimulated hSSCs osteogeneic differentiation apparent through increased mineralization expression of SPP1 and Alizarin Red staining, despite having reduced RUNX2 expression, an early osteogenesis marker (11).

The observed in vitro results in the Preciado study are likely because the exposure was a single, low-dose compared to other studies that use a single high-dose or fractionized doses that are needed therapeutically $(4,5,17,21,22)$. Other studies suggest SSC retention of stem cell characteristics is dose-dependent and can be altered with a single high-dose exceeding 10Gy (22-24). Schönmeyr et al. demonstrated the effects of highdose irradiation (7 and 12Gy) on rat SSCs (rSSCs) resulted in a dose dependent response compared to non-irradiated cells (22). After high-dose irradiation exposure rSSCs had a higher percentage of apoptotic cells and more cells in the $\mathrm{G}_{2}$ cellular arrest phase (22). The irradiated cells also had reduced expression of osteogeneic markers, $A L P$ and osteocalcin, as well as reduced expression of adipogenic markers, $L P L, C E B P \beta$, and Leptin 


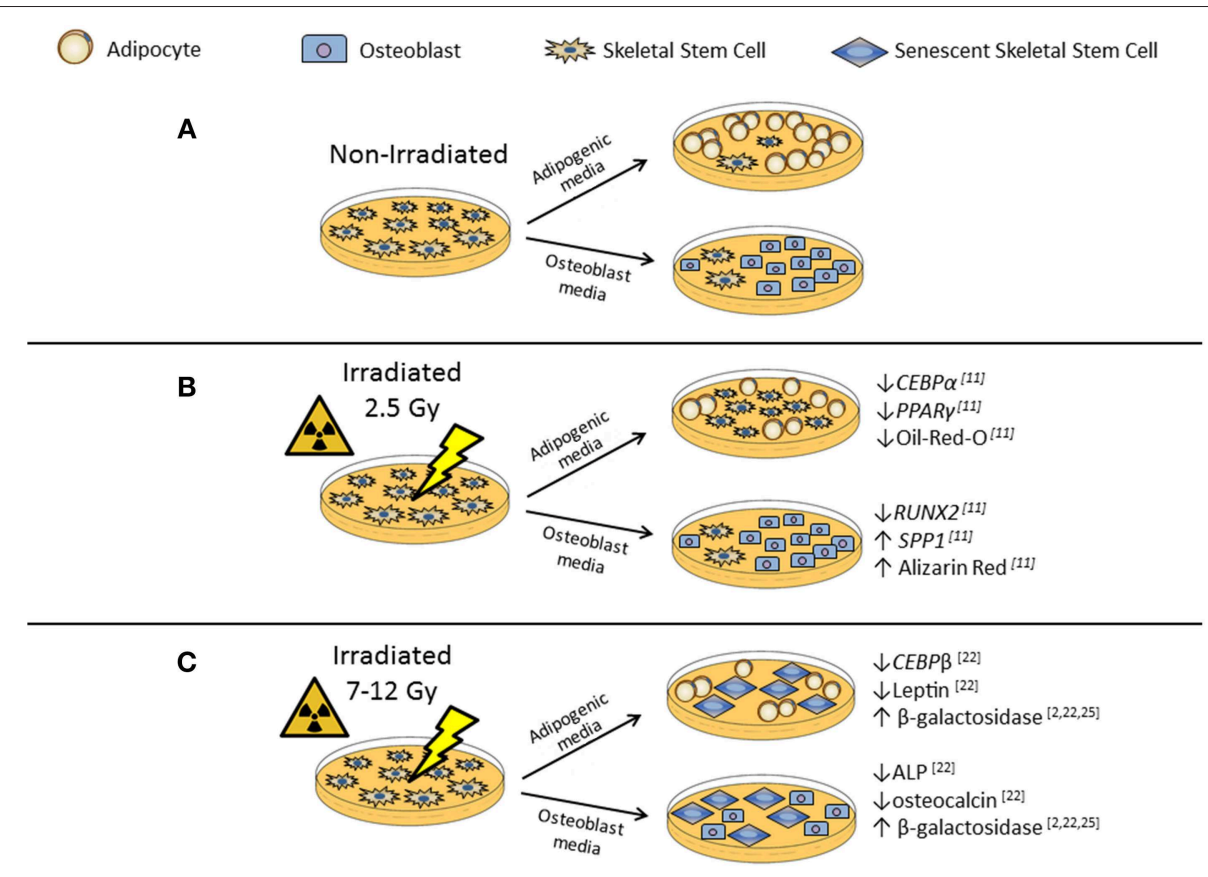

FIGURE 1 | In vitro irradiation affects adipogenic and osteogenic differentiation potential of skeletal stem cells (SSCs). (A) Non-irradiated SSCs, represent control differentiation. (B) Low-dose (2.5Gy) irradiated SSCs in adipogenic or osteogenic differentiation media have differing differentiation potentials. Low-dose irradiation caused reduced adipocyte differentiation with decreased adipocyte markers, such as CEBP $\alpha$ and PPAR $\gamma$, and decreased Oil-Red-O staining when compared to the control. Osteoblast differentiation showed decreased RUNX2 expression, but increased mineralization markers SPP1 and Alizarin Red staining after low-dose exposure when compared to the control. There was no significant difference in osteoblast differentiation when compared to the controls. (C) High-dose (7-12Gy) irradiated SSCs have reduced adipocyte and osteoblast differentiation potential and evidence of increased $\beta$-galactosidase activity, a marker for cellular senescence.

(22). The reduced differentiation potential seen in vitro could be evidence of irradiation-induced cellular senescence $(2,11)$. A marker for senescence, $\beta$-galactosidase, has been used in vitro to show irradiation-induced senescence in a time and dose dependent manner $(2,22,25)$. The level of differentiation potential down the osteogenic and adipogenic lineages of SSCs has been shown to be more sensitive or more resistant based on the dosage of ionizing radiation $(11,22,26,27)$. The altered SSC differentiation capacity impacts the hematopoietic niche and enhances engraftment of BM derived stem cell transplantations to the BM microenvironment (12).

Interestingly, irradiation induced by radionucleotides, such as Strontium-90, can also induce similar effects on SSCs (15). After 7 days of exposure in vitro, a pre-osteoblast cell line showed a decreased ability to proliferate, changes in cytokine expression, and changes in their ability to support hematopoietic progenitor proliferation and differentiation (15). Exposure to Strontium-90 also showed evidence of increased senescence through increased $\beta$-galactosidase as well as senescent morphology with enlarged cytoplasm and nucleus (15). Despite these intriguing findings in vitro, in vivo studies are necessary to determine if the same in vitro phenomena are observed.

\section{In vivo Irradiation in Rodents (Mouse and Rat) Cause Bone Loss and Increased BMAT}

In contrast to the decreased adipogenesis induced by irradiation observed in in vitro studies, in vivo models demonstrated that irradiation increases BMA and deteriorates trabecular bone at both high and low irradiation doses. For example, Willey et al. showed that as early as 3 days post low-dose (2Gy) whole-body irradiation of thirteen-week-old C57BL/6 mice there was a significant increase in osteoclast activity through significantly increased TRAP-5b serum levels and significantly increased osteoclast numbers per bone surface, although BMA analysis was not done (21). Ten days post low-dose (5Gy) whole-body irradiation exposure of $\mathrm{C} 57 \mathrm{BL} / 6$ mice resulted in rapid infiltration of BM adipocytes within the BM (5). This significant increase in BMA was coupled with a significant decrease in trabecular bone volume/total volume (BV/TV), which has been observed in 8 and 16-week-old C57BL/6 mice $(5,17)$. This irradiation-induced bone damage was not recovered 8 weeks post exposure $(5,17)$. BM recovery of 8 and 16week-old mice has been shown to be age and time dependent (17). Two and ten-days post irradiation exposure, the total number of BM cells in 8 and 16-week-old mice were significantly decreased by more than $60 \%$ (17). By 8 weeks, only 8 -weekold mice showed recovery to their BM cells (17). In sum, in vivo models using a wide range of irradiation doses have consistently shown that irradiation decreases trabecular bone volume and increases BMA compared to non-irradiated controls (Figure 2). This finding is likely due to a shift in SSC lineage differentiation (i.e., favoring adipogenesis over osteogenesis) that appears to be in response to irradiation-induced $\mathrm{BM}$ microenvironment alternations, rather than SSC autonomous responses to irradiation, because these same shifts are not observed in SSCs in vitro. 


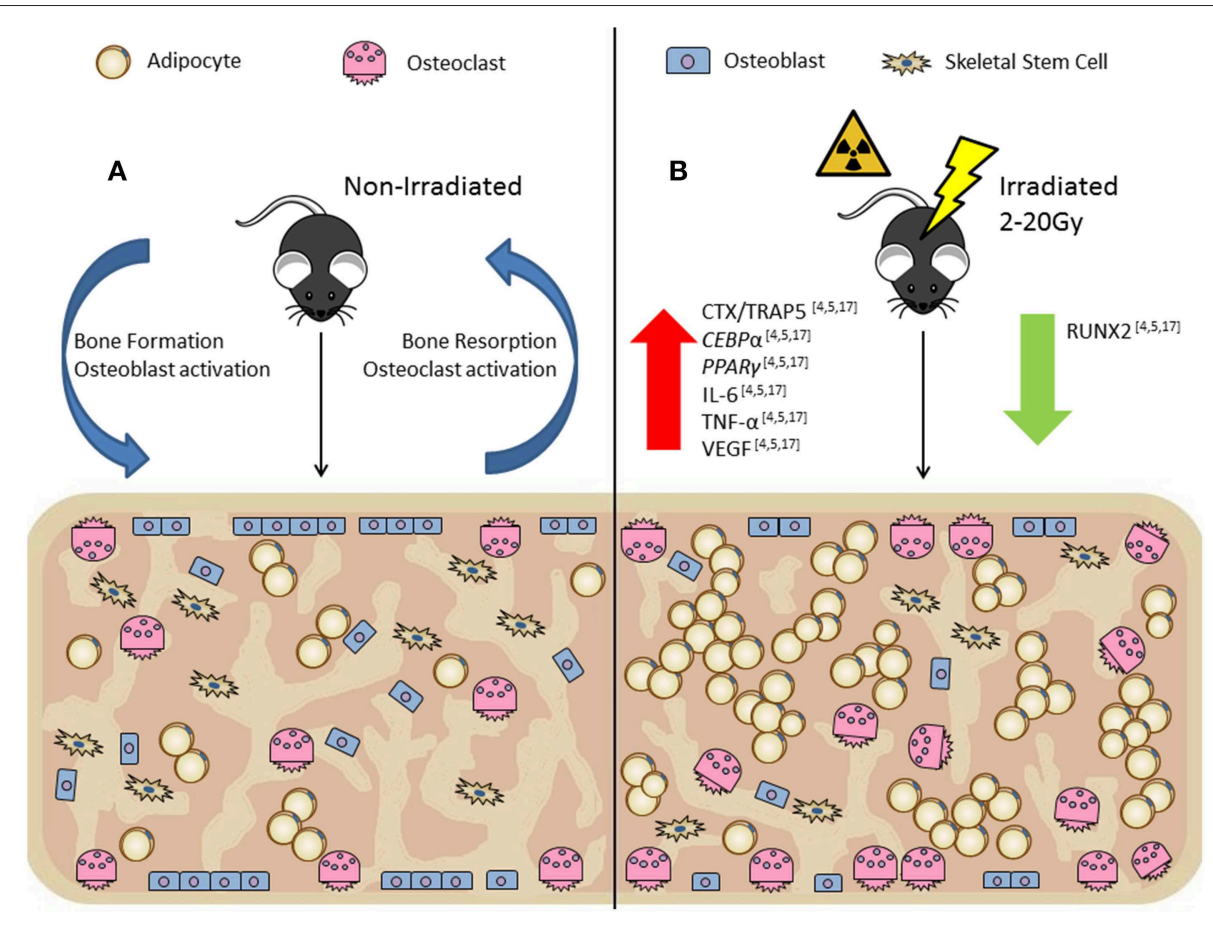

FIGURE 2 | In vivo rodent models show irradiation alters the bone marrow microenvironment by increasing osteoclast numbers per bone surface (Oc.S/BS) and decreasing osteoblast numbers per bone surface (Ob.S/BS) resulting in decreased trabecular bone volume with a rapid influx of bone marrow adipocytes.

(A) Non-irradiated control, demonstrates normal bone turnover processes. (B) Irradiated (2-20Gy), demonstrates the uncoupling of the bone formation/resorption ratio through increased CTXTRAP5 (osteoclast activity) and decreased RUNX2 (osteoblast activity) expression. In vivo irradiation exposure also has increased CEBP $\alpha$ and PPAR (adipogenesis markers), and IL-6, TNF- $\alpha$, and VEGF (inflammatory and senescent markers).

Localized irradiation has been shown to affect healthy tissue adjacent to the irradiation site, with about half of the localized dose being absorbed by healthy tissue and bone (4). In 4 month old male Sprague-Dawley rats, localized exposure of 20Gy to the right hind-limb, spanning the proximal tibia to the distal femur, revealed significant reductions in trabecular bone mineral density (tBMD) and trabecular BV/TV of the irradiated femur and also in the contralateral femur compared to the sham irradiated controls (4). Cortical thickness was not affected by irradiation, but cortical porosity was increased in the irradiated and contralateral femur (4). BMAT increased at 2 weeks and 12 weeks post-irradiation in the irradiated and contralateral tibias. These alterations in trabecular bone were due to a decrease in osteoblast surface per bone surface at 2 and 12 weeks post-irradiation in the irradiated and contralateral tibias (4). Within the BM, expression of RUNX2 and PPAR $\gamma$ of osteoblast and adipocyte progenitor cells were determined using reverse transcriptase-PCR (RT-qPCR) (4). At 2 weeks, RUNX2 and PPAR $\gamma$ expression were significantly decreased in both the irradiated and contralateral (4). By 12 weeks, the mRNA expression of RUNX2 continued to be downregulated by $94.5 \%$ in the irradiated and $44.1 \%$ in the contralateral, yet the expression of PPAR $\gamma$ was upregulated by 13 -fold in the irradiated and 9-fold in the contralateral relative to the control (4). This study demonstrated how irradiation-induced bone damage does not require direct exposure to result in impairment, referred to as the bystander effect $(4,28)$.
There is also a rapid increase in osteoclast activity after irradiation exposure, seen with an increase in osteocalcin and TRAP5 levels in rat serum (4). However, 2 weeks post-irradiation there were no significant effects on bone suggesting irradiation did not compromise or uncouple the bone formation/resorption ratio immediately after exposure (4). These results differ from the observed changes seen in many irradiation mouse models. By 12 weeks post-exposure, there were significant decreases in trabecular bone volume, yet osteoclastogenesis was now comparable to the controls while osteoblastogenesis was significantly decreased, resulting in an altered formation/resorption ratio within the BM microenvironment that affected bone quality (4).

\section{Clinical Trials Mirror in vivo Animal Model Findings}

Irradiation-induced bone loss has been reported to cause more than insufficiency fractures; other complications from irradiation therapy include osteitis and osteolysis (29). A patient study of 510 patients (ages 40-84 years) analyzed pelvic bone related complications after irradiation therapy for uterine cervical cancer. Osteolysis was detected in 4 patients and avascular necrosis of the femoral head was diagnosed in 2 patients post irradiation therapy (29). One-hundred patients were diagnosed with insufficiency fractures a median of $\sim 16.9$ months (range 1-87 months) after pelvic irradiation therapy (29). Of the patients diagnosed with insufficiency fractures, $85 \%$ had sacral involvement and $61 \%$ developed multiple pelvic insufficiency 
fractures; $40 \%$ of those patients had symmetric bilateral lesions of the sacral alae (29).

Patients can experience late stage complications from irradiation exposure as part of an advanced treatment for head and neck tumors (9). Osteoradionecrosis (ORN) of the jaw bones (and surrounding soft tissue) is the most severe last stage complication (9). ORN illustrates increased inflammation and the development of hypovascular, hypocellular, and hypoxic tissues, which causes increased cell death and collagen breakdown that exceeds the normal cell repair and collagen synthesis homeostasis $(9,30)$. ORN diagnostic criteria is a slow healing (failure to recover over a 3 month period) irradiationinduced necrosis of the bone, associated with surrounding tissue necrosis in the absence of local tumor necrosis, recurrence, or metastatic disease (9). Irradiated specimens were obtained from 40 patients treated for ORN (control specimens were obtained from non-irradiated patients treated from head and neck tumors) (9). The total irradiation dosage of these specimen ranged from 50.4 to $70.4 \mathrm{~Gy}$ (9). A histopathology examination on the bone and soft tissue samples revealed hyperemia and endarteritis as early effects of irradiation that were prolonged for up to 6 months post exposure (9). Signs of increased hypocellularity occurred rapidly after irradiation exposure; the irradiated bone samples showed greater cell loss than the soft tissue samples $(9,31)$. Evidence of thrombosis was apparent through densely fibrous material seen years post irradiation exposure $(9,31)$. There was a loss in vascular content, increase in BMAT, and fibrosis that showed a linear correlation to the time post exposure that were considered end stage markers of the irradiation-induced injury $(9,31)$. This current study suggested the increase in BMAT in the irradiated bone samples was due to the stunted bone turnover processes $(9,32)$.

Another clinical study showed 13 female patients, ages 35-63 years old, with gynecological malignancies that received irradiation or chemotherapy treatments had increased BMAT 6 and 12 months after initiating therapy treatments through repeated MRI scan (baseline, 6 months, and 12 months post therapy initiation) (33). Sagittal images of signal fat fraction (SFF) were taken in patients receiving focal irradiation therapy with the pelvic region as the target field (33). Approximately half of the irradiation therapy dose was absorbed by the sacrum and adjacent tissues and bones, such as the L 4 vertebral body (33). At the baseline MRI scan, the SFF of the BMAT in the L4 and S1 were similar, however, by the 6-month scan the increase in the SFF in the S1 was marginally higher than in L4 (33). The increased SFF seen in S1 compared to L4 correlated to the higher irradiation absorption at $\mathrm{S} 1$ resulting in more BMAT accumulation (33). It is believed there is a progressive conversion of the hematopoietic marrow to the more adipocyte rich, yellow marrow observed throughout these regions as a response to irradiation treatment (33). The SFF of the $\mathrm{L} 4$ and femoral neck increased at the 6-month post-treatment scan (irradiation and chemotherapy treatments combined in this analysis) (33). These significant increases of SFF in the L4 and femoral neck were compared to skeletal muscle and subcutaneous white adipose tissue as controls, demonstrating the effects of irradiation on localized tissues and more specifically on the BM microenvironment (33).

\section{Bisphosphonates and Osteoanabolic Agents Have Potential Restorative Effects on Irradiation-Induced Bone Damage}

Anti-resorptive agents, such as bisphosphonates (BPs), and osteoanabolic agents are commonly used as osteoporosis treatments. BPs mediate bone resorption through osteoclast apoptosis $(19,20,34)$. BPs can also reduce osteoblast and osteocyte apoptosis, but do not actively result in bone accrual (34). In rodent models, the administration of BPs following irradiation therapy can improve bone quality, bone strength, and $\operatorname{BMD}(19,35,36)$. However, there are conflicting data across patient trials regarding bone quality and pain management with BPs alone, in respect to cancer and irradiation therapies, suggesting combination treatments of BPs, and osteoanabolic agents may be needed to combat irradiation-induced bone damage (20, 35, 37-39).

Well-studied osteoanabolic treatments are human parathyroid hormone (hPTH) and sclerostin antibody (Scl$\mathrm{Ab}$ ). Both of these agents stimulate bone formation, but through different modes of action (40,41). Scl-Ab increases bone formation by inhibiting sclerostin binding to lipoprotein receptor protein (LRP) 5/6 that inhibits canonical WNT signaling and subsequently activating SSC differentiation into osteoblasts $(40,41)$. Scl-Ab also suppresses bone resorption through inhibitory effects on osteoclastogenesis regulators (41-43). hPTH stimulates both anabolic and catabolic activities with a net gain in favor of the former, but there is evidence that hPTH anabolic capabilities are dependent on its ability to stimulate osteoclastogenesis $(41,44)$. Not only do these osteoanabolic agents increase bone accrual, but they also decrease BMA (41). $\mathrm{Scl}-\mathrm{Ab}$ results in increased trabecular bone and decreased BMA, but hPTH has a direct effect on BMA reductions despite bone accrual (41). Since irradiation causes bone deterioration and adipocyte infiltration, which in combination may exacerbate bone related complications, administration of osteoanabolic agents in conjunction with irradiation therapy may prevent excessive bone loss and adipocyte infiltration (16).

\section{CONCLUSION}

Clinical studies and in vivo rodent models have shown highdose and sub-lethal dose irradiation causes rapid bone loss due to increased osteoclast activity and decreased osteoblast activity, which results in increased BMA and secondary late stage bone complications that are believed to be from continued irradiation damage (16). Through in vitro experiments, it has been shown that SSCs are capable of maintaining their proliferation, differentiation, and regenerative properties at lowdose irradiation exposure, but at a lower capacity than nonirradiated SSCs (11). However, after high-dose exposure SSCs lose their stem cell characteristics or experience cell death $(22-24,26,27)$. Currently, irradiation therapy is provided to patients in fractionized doses, but even low-doses over the course of several weeks show signs of decreased BMD and increased BMA (16). With decreased BMD, patients are at a greater risk for skeletal fractures and other bone related diseases and complications. A patient's quality of life is 
severely affected by these irradiation-induced bone incidences. Future directions point to more investigative research (and potential clinical practices) into the benefits of combination therapies to reverse the adverse side effects of irradiationinduced bone loss and adipocyte infiltration. Osteoanabolic agents, such as Scl-Ab or hPTH, and BPs may be needed in conjunction or following irradiation therapy treatments to combat the bone, tissue, and cell damages currently being observed.

\section{AUTHOR CONTRIBUTIONS}

SC wrote the manuscript. MR advised and edited the manuscript. $\mathrm{MR}$ and SC approved the final version of the manuscript.

\section{REFERENCES}

1. Bernier J, Hall EJ, Giaccia A. Radiation oncology: a century of achievements. Nat Rev Cancer. (2004) 4:737-47. doi: 10.1038/nrc1451

2. Chandra A, Park SS, Pignolo RJ. Potential role of senescence in radiation-induced damage of the aged skeleton. Bone. (2019) 120:42331. doi: 10.1016/j.bone.2018.12.006

3. Siegel RL, Miller KD, Jemal A. Cancer statistics, 2019., CA. Cancer J Clin. (2019) 69:7-34. doi: 10.3322/caac.21551

4. Zou Q, Hong W, Zhou Y, Ding Q, Wang J, Jin W, et al. Bone marrow stem cell dysfunction in radiation-induced abscopal bone loss. J Orthop Surg Res. (2016) 11:3. doi: 10.1186/s13018-015-0339-9

5. Green DE, Adler BJ, Chan ME, Lennon JJ, Acerbo AS, Miller LM, et al Altered composition of bone as triggered by irradiation facilitates the rapid erosion of the matrix by both cellular and physicochemical processes. PLOS ONE. (2013) 8:e64952. doi: 10.1371/journal.pone.0064952

6. Sparks RB, Crowe EA, Wong FC, Toohey RE, Siegel JA. Radiation dose distributions in normal tissue adjacent to tumors containing (131)I or (90)Y: the potential for toxicity. J Nucl Med. (2002) 43:1110-4.

7. Elgström E, Ohlsson TG, Eriksson SE. Cytokine evaluation in untreated and radioimmunotherapy-treated tumors in an immunocompetent rat model. Tumor Biol. (2017) 39:101042831769755. doi: 10.1177/10104283176 97550

8. Linard C, Marquette C, Mathieu J, Pennequin A, Clarençon D, Mathé D. Acute induction of inflammatory cytokine expression after gamma-irradiation in the rat: effect of an NF-kappaB inhibitor. Int J Radiat Oncol Biol Phys. (2004) 58:427-34. doi: 10.1016/j.ijrobp.2003.09.039

9. Curi MM, Cardoso CL, de Lima HG, Kowalski LP, Martins MD. Histopathologic and histomorphometric analysis of irradiation injury in bone and the surrounding soft tissues of the jaws. J Oral Maxillofac Surg. (2016) 74:190-9. doi: 10.1016/j.joms.2015.07.009

10. Delanian S, Lefaix JL. The radiation-induced fibroatrophic process: therapeutic perspective via the antioxidant pathway. Radiother Oncol. (2004) 73:119-131. doi: 10.1016/j.radonc.2004.08.021

11. Preciado S, Muntión S, Rico A, Pérez-Romasanta LA, Ramos TL, Ortega $\mathrm{R}$, Borrajo J, et al. Mesenchymal stromal cell irradiation interferes with the adipogenic/osteogenic differentiation balance and improves their hematopoietic-supporting ability. Biol Blood Marrow Transplant. (2018) 24:443-51. doi: 10.1016/j.bbmt.2017.11.007

12. Herberg S, Kondrikova G, Hussein KA, Periyasamy-Thandavan S, Johnson $\mathrm{MH}$, Elsalanty $\mathrm{ME}$, et al. Total body irradiation is permissive for mesenchymal stem cell-mediated new bone formation following local transplantation. Tissue Eng Part A. (2014) 20:3212-27. doi: 10.1089/ten.tea.2013.0663

13. Higashi T, Fisher SJ, Brown RS, Nakada K, Walter GL, Wahl RL. Evaluation of the early effect of local irradiation on normal rodent bone marrow metabolism using FDG: preclinical PET studies. J Nucl Med. (2000) 41:2026-35.

\section{FUNDING}

Funding was supplied by the NIH/NIGMS (P20GM121301). The authors' work was also supported by start-up funds from the Maine Medical Center Research Institute and the American Cancer Society (Research Grants \#IRG-16-191-33 and \#133077RSG-19-037-01-LIB). This research utilized services of the U54GM115516 Core, which was supported by NIH/NIGMS P20GM121301 (L. Liaw, PI), U54GM115516 (C. Rosen, PI), and P30GM106391 (R. Friesel, PI). Funding/research grants were provided by Amgen Inc. and UCB Inc. Amgen Inc. and UCB Inc. had no role in the study design, data collection/analysis, decision to publish, or preparation of the manuscript.

14. Nicolay NH, Lopez Perez R, Saffrich R, Huber PE. Radio-resistant mesenchymal stem cells: mechanisms of resistance and potential implications for the clinic. Oncotarget. (2015) 6:19366-80. doi: 10.18632/oncotarget.4358

15. Musilli S, Nicolas N, El Ali Z, Orellana-Moreno P, Grand C, Tack K, et al. DNA damage induced by Strontium-90 exposure at low concentrations in mesenchymal stromal cells: the functional consequences. Sci Rep. (2017) 7:41580. doi: 10.1038/srep41580

16. Sun $\mathrm{R}$, Zhu G, Wang J, Tong L, Zhai J. Indirect effects of X-irradiation on proliferation and osteogenic potential of bone marrow mesenchymal stem cells in a local irradiated rat model. Mol Med Rep. (2017) 15:370614. doi: 10.3892/mmr.2017.6464

17. Green DE, Adler BJ, Chan ME, Rubin CT. Devastation of adult stem cell pools by irradiation precedes collapse of trabecular bone quality and quantity. J Bone Miner Res. (2012) 27:749-59. doi: 10.1002/jbmr.1505

18. Ramchand SK, Seeman E. Advances and unmet needs in the therapeutics of bone fragility. Front Endocrinol. (2018) 9:505. doi: 10.3389/fendo.2018. 00505

19. Krempien R, Huber PE, Harms W, Treiber M, Wannenmacher M, Krempien B. Combination of early bisphosphonate administration and irradiation leads to improved remineralization and restabilization of osteolytic bone metastases in an animal tumor model. Cancer. (2003) 98:131824. doi: $10.1002 / \mathrm{cncr} .11646$

20. Arrington SA, Fisher ER, Willick GE, Mann KA, Allen MJ. Anabolic and antiresorptive drugs improve trabecular microarchitecture and reduce fracture risk following radiation therapy. Calcif Tissue Int. (2010) 87:26372. doi: 10.1007/s00223-010-9390-Z

21. Willey JS, Lloyd SAJ, Robbins ME, Bourland JD, Smith-Sielicki H, Bowman LC, et al. Early increase in osteoclast number in mice after whole-body irradiation with 2 Gy X rays. Radiat Res. (2008) 170:38892. doi: 10.1667/RR1388.1

22. Schönmeyr BH, Wong AK, Soares M, Fernandez J, Clavin N, Mehrara BJ. Ionizing radiation of mesenchymal stem cells results in diminution of the precursor pool and limits potential for multilineage differentiation. Plast Reconstr Surg. (2008) 122:64-76. doi: 10.1097/PRS.0b013e31817743cd

23. Nicolay NH, Sommer E, Lopez R, Wirkner U, Trinh T, Sisombath S, et al. Mesenchymal stem cells retain their defining stem cell characteristics after exposure to ionizing radiation. Int J Radiat Oncol. (2013) 87:11718. doi: 10.1016/j.ijrobp.2013.09.003

24. Li J, Kwong DLW, Chan GCF. The effects of various irradiation doses on the growth and differentiation of marrow-derived human mesenchymal stromal cells. Pediatr Transplant. (2007) 11:379-87. doi: 10.1111/j.1399-3046.2006.00663.x

25. Hong EH, Lee SJ, Kim JS, Lee KH, Um HD, Kim JH, et al. Ionizing radiation induces cellular senescence of articular chondrocytes via negative regulation of SIRT1 by p38 kinase. J Biol Chem. (2010) 285:128395. doi: 10.1074/jbc.M109.058628 
26. Mussano F, Lee KJ, Zuk P, Tran L, Cacalano NA, Jewett A, et al. Differential effect of ionizing radiation exposure on multipotent and differentiationrestricted bone marrow mesenchymal stem cells. J Cell Biochem. (2010) 111:322-32. doi: 10.1002/jcb.22699

27. Oliver L, Hue E, Séry Q, Lafargue A, Pecqueur C, Paris F, et al. Differentiationrelated response to DNA breaks in human mesenchymal stem cells. Stem Cells. (2013) 31:800-7. doi: 10.1002/stem.1336

28. Marín A, Martín M, Liñán $\mathrm{O}$, Alvarenga F, López M, Fernández L, et al. Bystander effects and radiotherapy. Reports Pract Oncol Radiother. (2015) 20:12. doi: 10.1016/j.rpor.2014.08.004

29. Kwon JW, Huh SJ, Yoon YC, Choi SH, Jung JY, Oh D, et al. Pelvic bone complications after radiation therapy of uterine cervical cancer: evaluation with MRI. Am J Roentgenol. (2008) 191:987-94. doi: 10.2214/AJR.07.3634

30. Marx RE. Osteoradionecrosis: a new concept of its pathophysiology. J Oral Maxillofac Surg. (1983) 41:283-8. doi: 10.1016/0278-2391(83)90294-X

31. Marx RE, Johnson RP. Studies in the radiobiology of osteoradionecrosis and their clinical significance. Oral Surgery Oral Med Oral Pathol. (1987) 64:379-90. doi: 10.1016/0030-4220(87)90136-8

32. Marx RE, Tursun R. Suppurative osteomyelitis, bisphosphonate induced osteonecrosis, osteoradionecrosis: a blinded histopathologic comparison and its implications for the mechanism of each disease. Int J Oral Maxillofac Surg. (2012) 41:283-9. doi: 10.1016/j.ijom.2011.12.016

33. Bolan PJ, Arentsen L, Sueblinvong T, Zhang Y, Moeller S, Carter JS, et al.Water-fat MRI for assessing changes in bone marrow composition due to radiation and chemotherapy in gynecologic cancer patients. J Magn Reson Imaging. (2013) 38:1578-84. doi: 10.1002/jmri.24071

34. Drake MT, Clarke BL, Khosla S. Bisphosphonates: mechanism of action and role in clinical practice. Mayo Clin Proc. (2008) 83:1032-45. doi: 10.4065/83.9.1032

35. Groenen KH, Pouw MH, Hannink G, Hosman AJ, van der Linden YM, Verdonschot N, et al. The effect of radiotherapy, and radiotherapy combined with bisphosphonates or RANK ligand inhibitors on bone quality in bone metastases. A systematic review. Radiother Oncol. (2016) 119:194201. doi: 10.1016/j.radonc.2016.03.001

36. Arrington SA, Damron TA, Mann KA, Allen MJ. Concurrent administration of zoledronic acid and irradiation leads to improved bone density, biomechanical strength, and microarchitecture in a mouse model of tumorinduced osteolysis. J Surg Oncol. (2008) 97:284-90. doi: 10.1002/jso.20949

37. Atahan L, Yildiz F, Cengiz M, Kaplan B, Özkan M, Yazici G, et al. Zoledronic acid concurrent with either high- or reduced-dose palliative radiotherapyin the management of the breast cancer patients with bone metastases: a phase IV randomized clinical study. Support Care Cancer. (2010) 18:6918. doi: 10.1007/s00520-009-0663-x

38. Rasmusson B, Vejborg I, Jensen AB, Andersson M, Banning AM, Hoffmann $\mathrm{T}$, et al. Irradiation of bone metastases in breast cancer patients: a randomized study with 1 year follow-up. Radiother Oncol. (1995) 34:17984. doi: 10.1016/0167-8140(95)01520-Q

39. Vassiliou V, Kalogeropoulou C, Christopoulos C, Solomou E, Leotsinides M, Kardamakis D. Combination ibandronate and radiotherapy for the treatment of bone metastases: clinical evaluation and radiologic assessment. Int J Radiat Oncol Biol Phys. (2007) 67:264-72. doi: 10.1016/j.ijrobp.2006. 08.022

40. Ominsky MS, Brown DL, Van G, Cordover D, Pacheco E, Frazier E, et al. Differential temporal effects of sclerostin antibody and parathyroid hormone on cancellous and cortical bone and quantitative differences in effects on the osteoblast lineage in young intact rats. Bone. (2015) 81:38091. doi: 10.1016/j.bone.2015.08.007

41. Costa S, Fairfield H, Reagan MR. Inverse correlation between trabecular bone volume and bone marrow adipose tissue in rats treated with osteoanabolic agents. Bone. (2019) 123:211-23. doi: 10.1016/j.bone.2019.03.038

42. Ominsky MS, Boyce RW, Li X, Ke HZ. Effects of sclerostin antibodies in animal models of osteoporosis. Bone. (2017) 96:63-75. doi: 10.1016/j.bone.2016.10.019

43. Spencer GJ. Wnt signalling in osteoblasts regulates expression of the receptor activator of NF B ligand and inhibits osteoclastogenesis in vitro. J Cell Sci. (2006) 119:1283-96. doi: 10.1242/jcs.02883

44. Sims NA, Vrahnas C. Regulation of cortical and trabecular bone mass by communication between osteoblasts, osteocytes and osteoclasts. Arch Biochem Biophys. (2014) 561:22-8. doi: 10.1016/j.abb.2014.5.015

Conflict of Interest Statement: The authors declare that the research was conducted in the absence of any commercial or financial relationships that could be construed as a potential conflict of interest.

Copyright (c) 2019 Costa and Reagan. This is an open-access article distributed under the terms of the Creative Commons Attribution License (CC BY). The use, distribution or reproduction in other forums is permitted, provided the original author(s) and the copyright owner(s) are credited and that the original publication in this journal is cited, in accordance with accepted academic practice. No use, distribution or reproduction is permitted which does not comply with these terms. 